\title{
LA BIOPSIA DE PIEL EN LEPRA
}

\author{
GERZAIN RODRIGUEZ, M.D.*
}

La biopsia cutánea es un procedimiento esencial en el estudio del paciente con lepra porque confirma definitivamente la sospecha clínica, clasifica la enfermedad, contribuye a evaluar los resultados del tratamiento y establece los diagnósticos diferenciales (1). Estos criterios son especialmente útiles en los niños, en quienes los cambios sensitivos son difíciles de evaluar, y en las lesiones incipientes (1). De igual manera, la biopsia puede dar información de primera mano sobre los estados reaccionales. La selección de la zona de la cual se va a tomar la biopsia es muy importante, y como es la regla para la mayoría de las enfermedades que afectan la piel, debe tomarse de una lesión típica, plenamente constituída (2). Debe hacerse del borde activo hacia el centro de la lesión y no es necesario extenderse hacia la piel normal. Si existen lesiones diferentes es conveniente tomar varias biopsias (2-3).

El diagnóstico histopatológico de la lepra se basa no sólo en el tipo y distribución de los infiltrados granulomatosos sino principalmente en los cambios histopatológicos que se producen en los filetes nerviosos. (Figs. 1-2). A su vez, los nervios más comúnmente afectados son los filetes dérmicos profundos y subcutáneos, (Figs. 1-2), así como los troncos nerviosos superficiales, o los que se apoyan sobre protuberancias óseas, más sujetos al frío y al trauma.

Por lo anterior, se deduce que es indispensable que la biopsia sea amplia y profunda para que incluya filetes nerviosos donde se presentan los cambios diagnósticos. (Figs. 1-2). El tamaño ideal de la biopsia debe ser de $5 \mathrm{~mm}$ de ancho, de $10 \mathrm{~mm}$ de longitud, incluyendo la unión dermo-hipodérmica, y de más de $7 \mathrm{~mm}$ de profundidad para que haya hipodermis en la muestra. (Fig. 1). Los sacabocados de $6 \mathrm{~mm}$ o menores son útiles en las lesiones lepromatosas obvias pero son inadecuados para las lesiones incipientes o en las que hay controversia o dificultad en la clínica. La presencia de hipodermis suficiente es mandatoria en los casos de reacción leprosa, principalmente en el eritema nodoso leproso, que tiene los cambios típicos en el panículo adiposo.

Existen casos raros de lepra neural pura o primaria, en los que no hay lesiones cutáneas, pero se presentan los cambios sensitivos, neuríticos, tróficos o motores que indican el daño neural. La selección del sitio de la biopsia cuando exista esta sospecha clínica debe ser de un área de cambios máximos en la sensibilidad y tomando ampliamente la hipodermis para ver allí los cambios neurales de la lepra. (Fig. 2). Sólo cuando este procedimiento falle se debe recurrir a la biopsia neural.

Una vez tomada la biopsia puede colocarse sobre papel blanco, firme, que puede ser un cartón o cartulina cualquiera, para evitar que se entorche, hecho que dificulta su orientación durante el proceso. Se coloca en el fijador, que preferiblemente debe ser formol tamponado ar $10 \%$ en la proporción de 20 volúmenes de fijador por cada volumen de la biopsia (20:1). La fórmula del formol tamponado es la siguiente:

$\begin{array}{lcc}\text { Formalina al 37-40\% } & 100 & \mathrm{ml} \\ \text { Agua destilada } & 900 & \mathrm{ml} \\ \text { Fosfato sódico monobásico } & 4 & \mathrm{~g} \\ \text { Fosfato sódico dibásico } & 6.5 & \mathrm{~g}\end{array}$

La solución de formaldehído (gas formaldehído en agua, aproximadamente al $40 \%$, llamado formalina) se toma como solución al $100 \%$ cuando se elaboran formoles con otro porcentaje. Ejemplo: $10 \mathrm{ml}$ de formalina $+90 \mathrm{ml}$ de agua $=$ formol al $10 \%$.

*Jefe del Grupo de Patología, Instituto Nacional de Salud. A.A.80334, Bogotá. Profesor Titular de Cátedra de los Departamentos de Morfología y Patología de la Facultad de Medicina de la Universidad Nacional. Bogotá. 


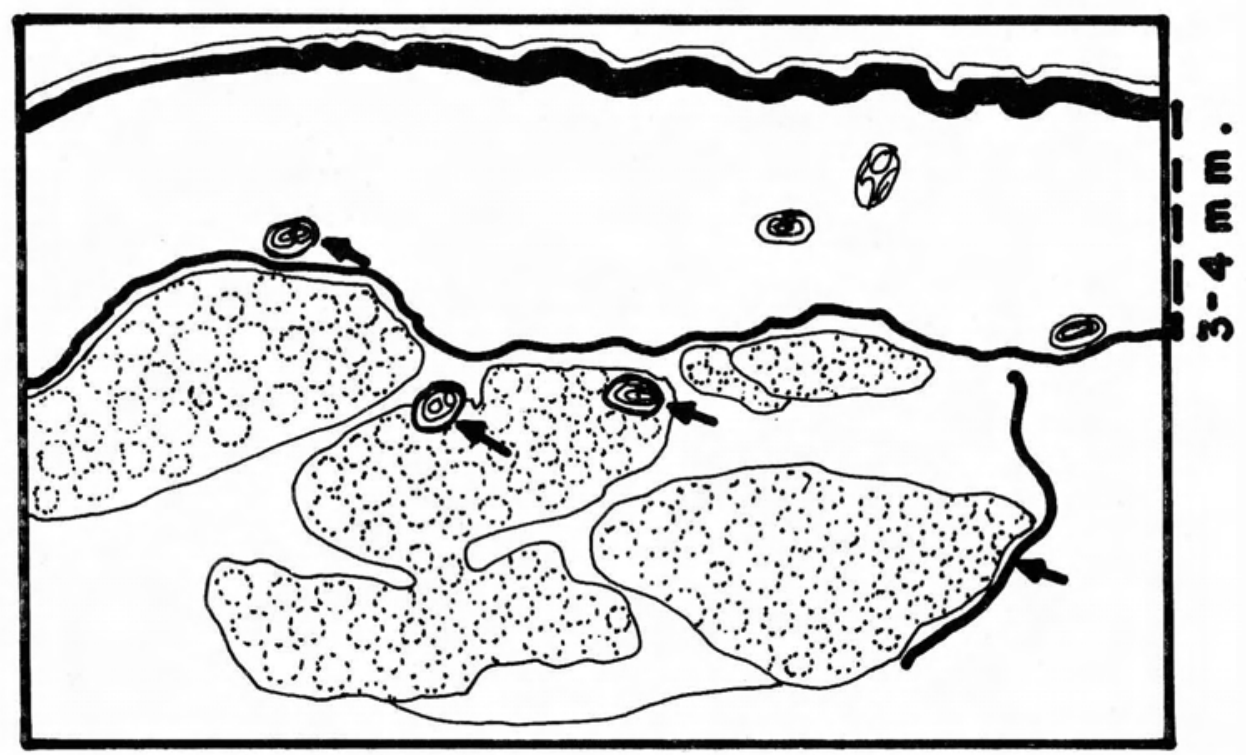

Fig. 1. Esquema de la biopsia adecuada para estudiar la lepra. Las flechas apuntan hacia nervios del límite dermohipodérmico y del subcutis, cuyo análisis es esencial para el diagnóstico. Nótese que el mayor grosor dérmico, en el tronco y el muslo, es apenas de 3-4 $\mathrm{mm}$ y que por lo tanto la profundidad de la biopsia debe ser mayor de estas dimensiones para que incluya tejido hipodérmico representativo.

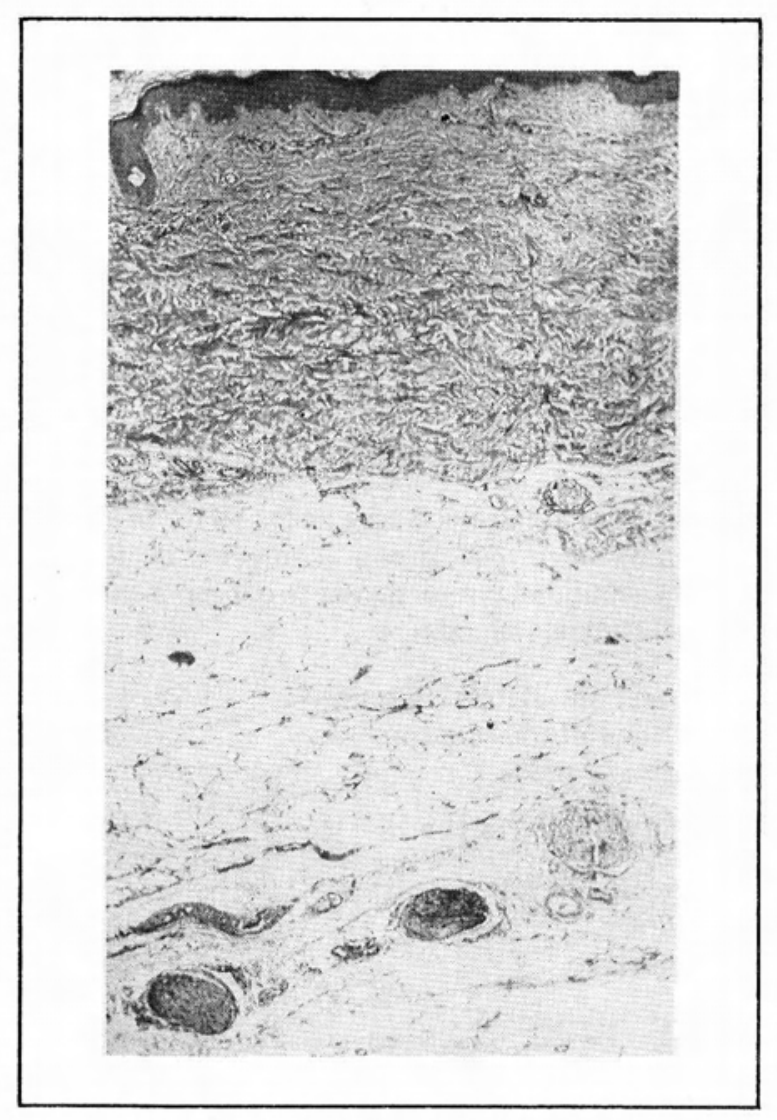

Si no se dispone de este fijador se pueden utilizar los siguientes substitutos:

\section{Formol salina}

Formalina al $37-40 \%$

Cloruro de sodio

Agua corriente

$\begin{array}{rr}100 & \mathrm{ml} \\ 9 & \mathrm{~g} . \\ 900 & \mathrm{ml}\end{array}$

\section{Formol al $10 \%$}

$\begin{array}{lll}\text { Formalina al } 37-40 \% & 10 & \mathrm{ml} \\ \text { Agua corriente } & 90 & \mathrm{ml}\end{array}$

Es importante no usar formol puro, que endurece excesivamente la muestra y no permite cortarla con el micrótomo. El frasco en que se coloca la biopsia debe ser de boca ancha y estar limpio, idenficado claramente con el nombre del paciente.

La solicitud de examen histopatológico debe contener los datos clínicos pertinentes para establecer la

Fig. 2. Nervios del límite dermohipodérmico y de la hipodermis con cambios proliferativos e inflamatorios típicos de lepra. Nótese la ausencia de inflamación en otras áreas de la dermis o de la hipodermis. Lepra Neural HE 40 X. 
correlación clinicohistopatológica adecuada, tales como edad, sexo, raza, sitio de donde se toma la biopsia, número y descripción básica de las lesiones, presencia de anestesia al calor, al frío y al dolor, tratamientos previos y antecedentes personales y familiares de importancia. La biopsia de piel es una interconsulta como cualquiera otra, cuyo resultado se basa no sólo en hallazgos descriptivos de la lámina histológica sino en la historia del paciente.

El espécimen se describe microscópicamente en el laboratorio, anotando si es un "punch" o una elipse, el tamaño del mismo y la presencia o no de hipodermis. Se procesa para inclusión en parafina y se hacen 2 3 láminas teñidas con $\mathrm{HE}$, con cortes seriados y una o más láminas teñidas con $\mathrm{ZN}$, también con cortes seriados, según la técnica abajo mencionada (5). Una coloración adicional, opcional, es la tricrómica de Masson, que permite demostrar bien los nervios o remanentes de los mismos, hecho que ayuda al diagnóstico principalmente en las formas tuberculoides (Figs. 3-4).

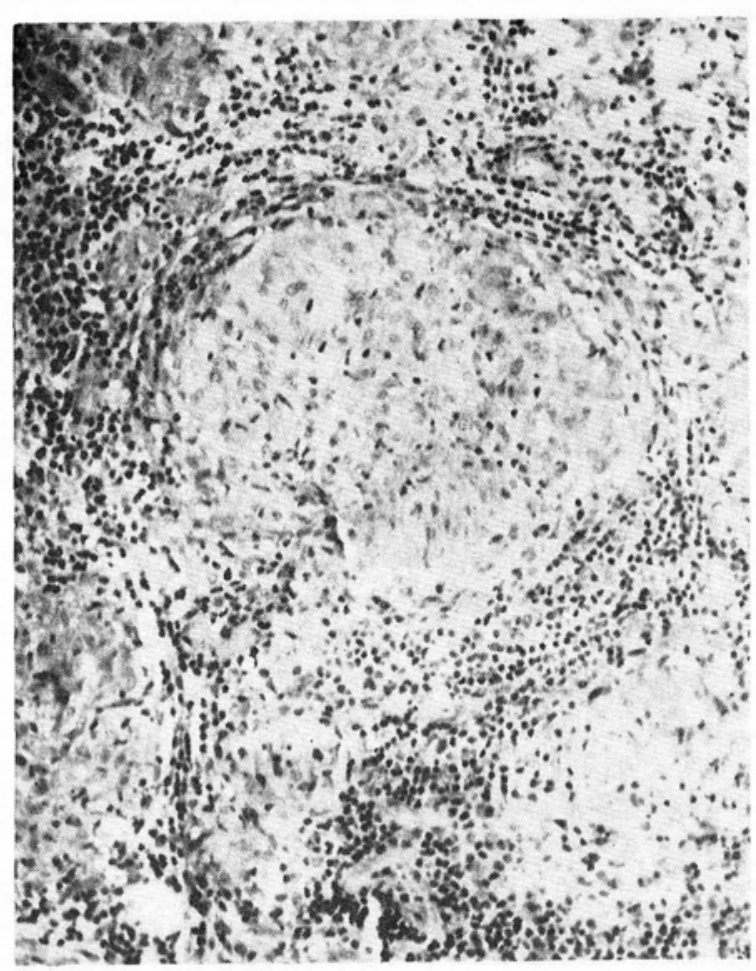

Fig. 3. Granuloma epiteloide en LT. La imagen no permite identificar remanentes del nervio. HE $160 \mathrm{X}$.

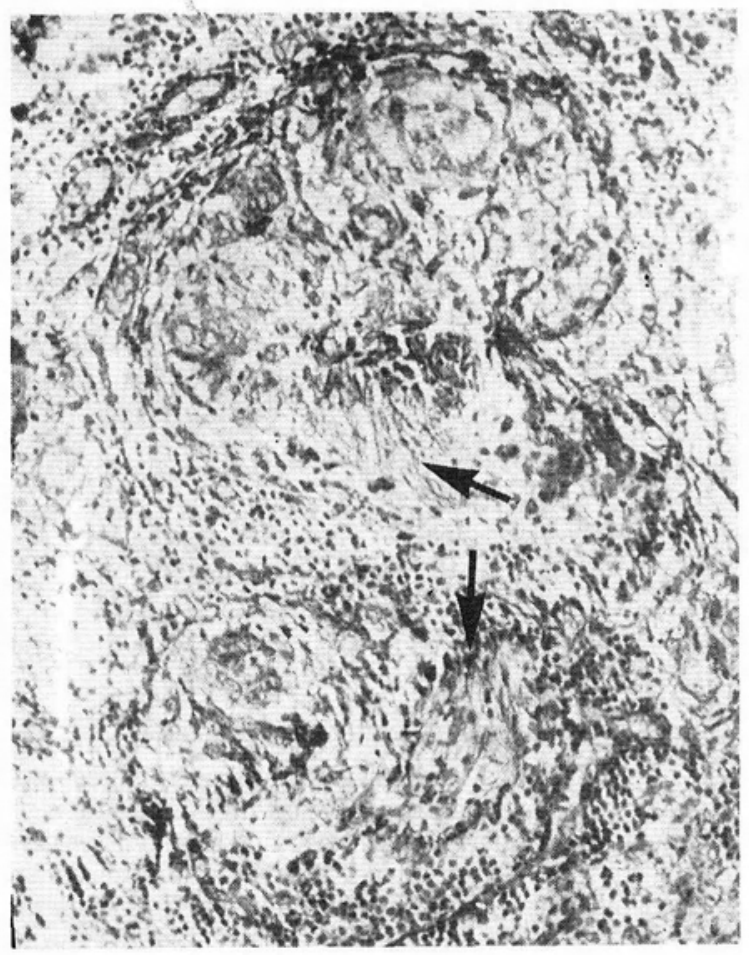

Fig. 4. Corte seriado de la misma lesión de la figura 3 , teñido con tricrómico. Se identifican claramente remanentes neurales en el centro del granuloma, que se tiñen de azul. (Flechas) Tricrómico $160 \mathrm{X}$.

Coloración de Z.N. utilizada en el INS.

\section{Reactivos}

$\begin{array}{lrl}\text { 1. Fucsina básica } & 1 & \mathrm{~g} \\ \text { Alcohol absoluto } & 10 & \mathrm{ml} \\ \text { Fenol al 5\% en agua destilada } & 100 & \mathrm{ml}\end{array}$

Disuelva la fucsina básica en $10 \mathrm{~s} 10 \mathrm{ml}$ de alcohol absoluto; luego mezcle con la solución de fenol al $5 \%$. (Estabilidad por un mes si se conserva en frasco herméticamente tapado).

2. Acido sulfúrico al $5 \%$ acuoso

Acido sulfúrico concentrado $\quad 5 \mathrm{ml}$

Agua destilada

$95 \mathrm{ml}$

3. Hematoxilina de Harris

4. Mezcla Xilol-Petrolato*

Xilol

2 partes

Petrolato

1 parte

*El Petrolato (Parafina líquida fluida ${ }^{R}$, Merck, Cat. 7174), es parafina liquida a temperatura ambiente. Se puede substituir por aceites comerciales comestibles tales como Oleo-Soya, Mazorca Oro, Premier, Oleocali, etc. 


\section{Procedimiento}

1. Cortes de 5 micras.

2. Llevar los cortes al horno, a $60^{\circ} \mathrm{C}$ durante 30 minutos.

3. Remover la parafina, en la mezcla Xilol-Petrolato durante 15 minutos, a temperatura ambiente.

4. Dejar escurrir y secar con papel secante.

5. Colocar los cortes en un bastidor de coloración y filtrar la fucsina carbólica sobre el tejido, inundando la laminilla entera.

6. Calentar la lámina con un mechero, hasta que la solución comience a emitir vapores. No permitir que la solución hierva puesto que el colorante se precipita.

7. Dejar actuar la solución de fucsina carbólica por 20 minutos a temperatura ambiente.

8. Lavar en agua corriente por 5 minutos.

9. Decolorar con ácido sulfúrico acuoso al $5 \%$ por 4 minutos.

10. Lavar en agua corriente por 5 minutos.

11. Agregar hematoxilina de Harris como colorante de contraste, por 2 minutos.

12. Lavar en agua corriente.

13. Diferenciar con agua amoniacal por 5 segundos.

14. Lavar en agua corriente.

15. Dejar secar a temperatura ambiente y aclarar con 2 cambios en Xilol.

16. Montar con Permount.

En la descripción microscópica de la biopsia no se debe omitir la mención del estado de los nervios. Se debe afirmar si la lepra es pauci o multibacilar y la variedad dentro de la clasificación de Ridley-Jopling (3-4). Las formas paucibacilares son aquellas en las que no se demuestra ningún bacilo. Esta descripción es esencial por cuanto de ella depende el tratamiento que recibirá el paciente. Debe correlacionarse con los resultados de la baciloscopia y si hubiera discrepancia notoria entre los dos, debe repetirse la baciloscopia, pues para los efectos actuales del Programa de Control de la Lepra, es éste procedimiento el más fácil y accesible para determinar si el paciente tiene una forma pauci o multibacilar de la enfermedad.
Términos como "compatible" o "sugestiva", si bien a veces son ineludibles, no deben tener cabida en el informe o se deben restringir al máximo. En general, se recurre a ellos cuando la biopsia es tan pequeña que no permite el análisis de los nervios. Indican probabilidad y que hay duda en el patólogo; por lo tanto, no deben tomarse como afirmaciones de certeza. En casos de duda debe recurrirse a otros exámenes o a nuevas biopsias, sin olvidar jamás la sabia recomendación de la OMS: "Si hay un diagnóstico que no debe formularse sin tener la absoluta certeza, es el de la lepra. Si existe la más mínima duda en el diagnóstico, el enfermo debe mantenerse en observación hasta que nuevos datos confirmen la enfermedad. Esto evitará el daño psicológico, social y de otro tipo que podría causársele innecesariamente en caso de diagnóstico equivocado". (Guía para la lucha antileprosa - Organización Mundial de la Salud, Ginebra, 1980).

\section{AGRADECIMIENTO}

A los doctores Luis Carlos Orozco y Orlando Ricaurte por comentarios y críticas; al señor Enrique Rivera por el trabajo fotográfico; a la señora Beatriz Mejía por el esquema de la figura 1, basado en fotografías de lesiones originales y a la señora Blanca $\mathrm{H}$. Carrillo de Lara por mecanografiar el manuscrito.

\section{BIBLIOGRAFIA}

1. Rodríguez G. Problemas diagnósticos en la lepra inicial. Acta Méd Col 1979; 4 (3): 113-118.

2. Rodríguez G, Jaramillo F, Chalela JG. La biopsia de piel. Biomédica, 1987; 7 (1-2): 42-64.

3. Ridley DS. Skin biopsy in leprosy. Documena Geigy. 1977, Ciba-Geigy. Basilea - Suiza.

4. Ridley DS. Histological classification and the inmunological spectrum of leprosy. Bull. WHO, 1974; 51: 451-465.

5. Naranjo P de, Rodríguez G, Rodríguez J, Caldas M. La Coloración de Ziehl-Neelsen en Histopatología - Biomédica, 1988; 8 (3-4). 